\title{
Case Report \\ Use of Contrast-Enhanced MR Angiography (CE-MRA) for the Diagnosis of a Vascular Ring Anomaly in a Dog
}

\author{
Silke Hecht, April M. Durant, William H. Adams, and Gordon A. Conklin \\ Department of Small Animal Clinical Sciences, C247 Veterinary Medical Center, \\ University of Tennessee College of Veterinary Medicine, 2407 River Dr., Knoxville, TN 37996, USA
}

Correspondence should be addressed to Silke Hecht, shecht@utk.edu

Received 28 June 2012; Accepted 1 August 2012

Academic Editors: C. Hyun and G. Mazzullo

Copyright $\odot 2012$ Silke Hecht et al. This is an open access article distributed under the Creative Commons Attribution License, which permits unrestricted use, distribution, and reproduction in any medium, provided the original work is properly cited.

\begin{abstract}
A 4-month-old female mixed breed dog was presented to the University of Tennessee College of Veterinary Medicine with a history of regurgitation and cachexia. Thoracic radiographs revealed focal megaesophagus cranial to the heart base. Magnetic resonance imaging (MRI) was performed. True fast imaging with steady-state precession (TrueFISP), fast low angle shot (FLASH), and short tau inversion recovery (STIR) sequences were acquired prior to contrast medium administration. Contrast-enhanced magnetic resonance angiography (CE-MRA) demonstrated focal megaesophagus and position of the aortic arch to the right of the esophagus. A small ductus diverticulum and an indistinct linear soft tissue band crossing the esophagus were also noted. Surgical exploration confirmed MR diagnosis of a persistent right aortic arch (PRAA) with left ligamentum arteriosum. The dog improved following surgery but was unable to be transitioned to dry food. To our knowledge this is the first report describing the use of CE-MRA for preoperative diagnosis and guided surgical treatment of a vascular ring anomaly in a dog.
\end{abstract}

\section{Introduction}

Persistent right aortic arch (PRAA) is the most common vascular ring anomaly in dogs, but other types have been reported and may require a different surgical approach [1]. A definitive diagnosis prior to surgical intervention is desirable to minimize prolonged anesthesia and associated complications. This report describes the successful preoperative diagnosis of a PRAA with left ligamentum arteriosum by means of contrast-enhanced MR angiography (CE-MRA).

\section{Case Presentation}

A 4-month-old female mixed breed dog, weighing $6.1 \mathrm{~kg}$, was referred to the University of Tennessee College of Veterinary Medicine for evaluation of regurgitation and poor body condition despite a ravenous appetite. A minimum database prior to referral included a complete blood count $(\mathrm{CBC})$ and biochemical profile. There were no significant abnormalities noted on CBC. Biochemical abnormalities included hyperphosphatemia $(8.4 \mathrm{mg} / \mathrm{dL}$, reference range
2.9-6.6 mg/dL), hyperglycemia $(114 \mathrm{mg} / \mathrm{dL}$, reference range $60-110 \mathrm{mg} / \mathrm{dL})$, hypoproteinemia $(5.2 \mathrm{~g} / \mathrm{dL}$, reference range $5.4-8.2 \mathrm{~g} / \mathrm{dL}$ ), and hypoglobulinemia $(1.5 \mathrm{~g} / \mathrm{dL}$, reference range $2.3-5.2 \mathrm{~g} / \mathrm{dL}$ ). On presentation the dog was bright and alert with a body condition score of $2 / 5$. All vital parameters were normal.

Right lateral and ventrodorsal thoracic radiographs were obtained. A dilated mostly gas-filled structure depressing the trachea ventrally consistent with focal megaesophagus was noted cranial to the cardiac silhouette on the right lateral view (Figure 1(a)). On the VD view, there was moderate uniform widening of the cranial mediastinum with the aortic arch located within the right aspect of the cranial mediastinum and the trachea located on midline and deviated to the left at the level of the aortic arch (Figure 1(b)). No additional abnormalities were detected.

MRI was performed using a 1.0 Tesla MR system (Magnetom Harmony; Siemens Medical Solutions, Malvern, PA, USA) to further characterize the suspected vascular ring anomaly. Pre- and postcontrast (Magnevist, Bayer HealthCare Pharmaceuticals Inc., Wayne, NJ, USA; $0.2 \mathrm{mmol} / \mathrm{kg}$ 


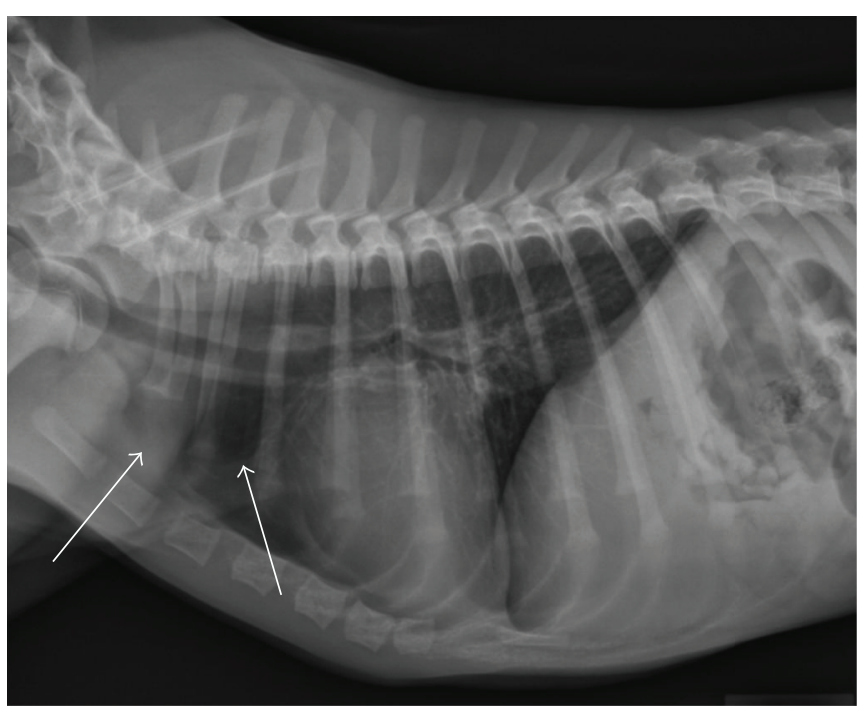

(a)

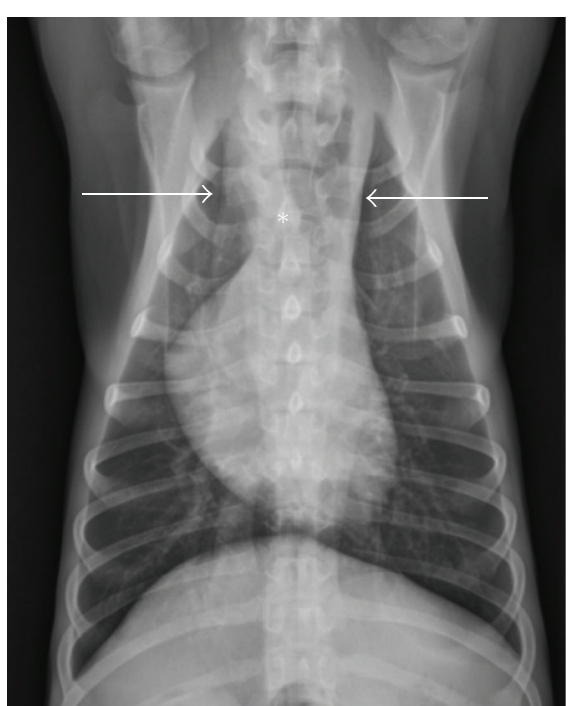

(b)

FIGURE 1: Right lateral (a) and ventrodorsal (b) radiographs of the thorax. A dilated mostly gas-filled structure (arrows) consistent with focal megaesophagus is present cranial to the cardiac silhouette on the lateral view (a). On the VD view (b) the aortic arch is located within the right aspect of the cranial mediastinum $(*)$, the trachea is displaced to the left at the level of the aortic arch, and there is mild widening of the cranial mediastinum (arrows).

TABLE 1: Magnetic resonance imaging parameters.

\begin{tabular}{|c|c|c|c|c|c|c|}
\hline Pulse sequence & $\begin{array}{l}\text { Slice thickness } \\
(\mathrm{mm})\end{array}$ & $\begin{array}{c}\text { Time of repetition } \\
(\mathrm{ms})\end{array}$ & $\begin{array}{l}\text { Time of echo } \\
(\mathrm{ms})\end{array}$ & $\begin{array}{c}\text { Number of } \\
\text { averages }\end{array}$ & $\begin{array}{c}\text { Time of inversion } \\
(\mathrm{ms})\end{array}$ & Flip angle \\
\hline Transverse TrueFISP & 5 & 6.8 & 3.4 & 2 & \multirow{7}{*}{145} & 70 \\
\hline Dorsal TrueFISP & 3 & 8.72 & 4.36 & 2 & & 70 \\
\hline Sagittal TrueFISP & 3 & 8.72 & 4.36 & 2 & & 70 \\
\hline Transverse FLASH 2D & 4 & 433 & 7.49 & 1 & & 70 \\
\hline Transverse T2 STIR & 5 & 7070 & 106 & 1 & & 150 \\
\hline $\begin{array}{l}\text { Immediate postcontrast sagittal FLASH } 3 \mathrm{D} \\
\text { (CE-MRA) }\end{array}$ & 1 & 5.25 & 2.29 & 1 & & 30 \\
\hline $\begin{array}{l}\text { Delayed postcontrast dorsal FLASH 3D } \\
\text { (CE-MRA) }\end{array}$ & 1.5 & 6.41 & 3.38 & 1 & & 10 \\
\hline
\end{tabular}

TrueFISP: true fast imaging with steady-state precession; FLASH: fast low angle shot; STIR: short tau inversion recovery; CE-MRA: contrast enhancedmagnetic resonance angiography.

IV) sequences were obtained, including true fast imaging with steady state precession (TrueFISP), fast low angle shot (FLASH 2D); T2 STIR, and contrast-enhanced angiography (CE-MRA) (immediate and delayed FLASH 3D) (Table 1). The cranial thoracic esophagus was moderately dilated with fluid and gas. The aortic arch and cranial descending aorta were located along the right aspect of the trachea and esophagus (Figure 2(a)), resulting in leftward displacement of these structures. A thin linear band of tissue was present extending from the cranial descending aorta leftward immediately caudal to the level of maximum esophageal dilation (Figure 2(b)). On maximum intensity projection (MIP) of CE-MRA data, there was no evidence of blood flow through the linear structure; however, there was a small focal dilation of the proximal descending aorta consistent with a ductus diverticulum (Figure 2(c)). 3D reconstructed images of CEMRA data were less helpful than anticipated due to inability to visualize nonvascular structures such as trachea and esophagus and resultant difficulties in anatomic orientation. An MR diagnosis of persistent right aortic arch with left ligamentum arteriosum and focal megaesophagus was made, and the owners opted for surgical correction.

The patient was placed in right lateral recumbence and prepared for a left thoracotomy. A left fifth thoracotomy was performed and a persistent right aortic arch with left ligamentum arteriosum was confirmed surgically. The mediastinum was bluntly dissected from the left lateral wall of the esophagus in the region of the stricture. The ligamentum arteriosum was bluntly dissected and double ligated. An oroesophageal tube was passed through the strictured region 


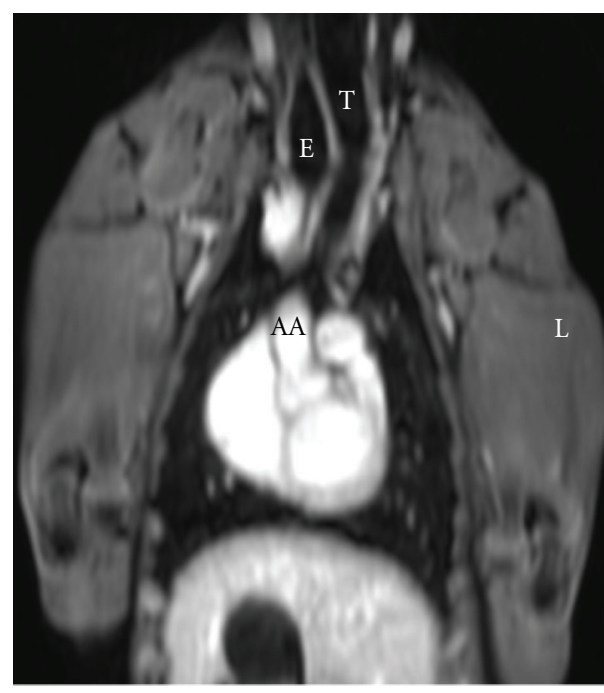

(a)

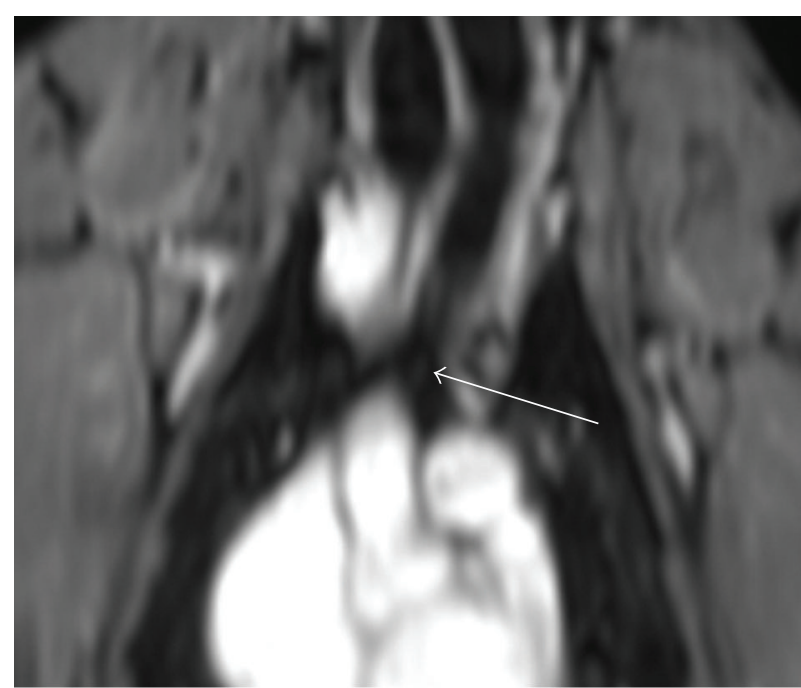

(b)

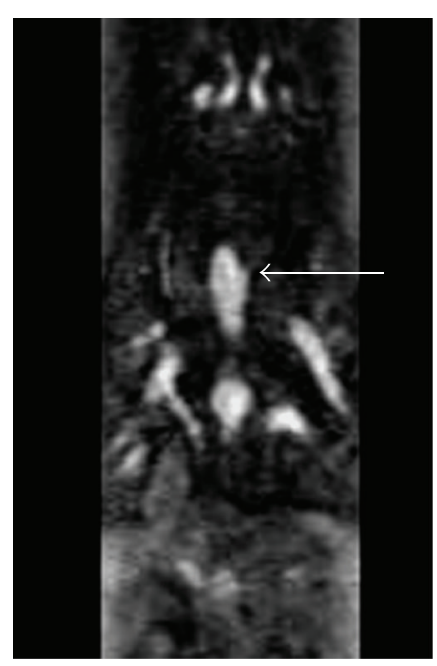

(c)

Figure 2: CE-MRA images demonstrating persistent right aortic arch with left-sided ligamentum arteriosum and focal megaesophagus. Dorsal plane image (a) shows the location of the aortic arch (AA) on the right and displacing the trachea (T) and dilated esophagus (E) to the left. An indistinct linear soft tissue band extends leftward from the proximal descending aorta immediately caudal to the area of maximum esophageal dilation ((b); arrow) consistent with the left ligamentum arteriosum. Dorsal maximum intensity projection reconstructed image at the level of the cranial descending aorta (c) demonstrates a small focal left-sided dilation of the aorta consistent with the ductus diverticulum (arrow). There is no evidence of blood flow through the ligamentum arteriosum.

to identify any remaining fibrous bands of the ligamentum arteriosum. The thoracotomy was closed routinely. Postsurgical recovery was uneventful. The following day blenderized food was offered in a raised position, and the patient was held in an upright position for 15 minutes after eating. The dog had an excellent appetite and did not exhibit any signs of regurgitation while in the hospital. The owner was instructed to continue elevated feedings with a blenderized diet for 24 weeks, with a gradual taper to a dry diet. Instructions to gradually decrease the height of the food bowl were also provided.

A telephone interview with the owner was conducted 8 weeks following surgery. The patient had gained weight, however, regurgitation resumed during attempts to transition to a dry diet. Follow-up radiographs by the referring veterinarian confirmed continued focal megaesophagus. The patient is currently maintained and asymptomatic on a mash diet that is fed slightly elevated.

\section{Discussion}

Persistent right aortic arch (PRAA) is a vascular ring anomaly where the right 4 th arch, rather than the left 4 th, is retained to form the aorta [1]. The presence of a left ligamentum arteriosum, connecting the pulmonary artery to the right aortic arch, results in compression of the esophagus which in turn 
leads to retention of food and esophageal dilation. The most common clinical signs related to a PRAA are regurgitation, poor body condition, and occasionally coughing, stridor and dyspnea [1]. Clinical signs tend to be noted during the weaning period, and regurgitation occurs shortly after the introduction of solid foods.

PRAA accounts for $95 \%$ of all cardiovascular ring anomalies [1-3]. Other vascular ring anomalies include double aortic arch, persistent right ductus arteriosus, aberrant left subclavian artery, aberrant right subclavian artery, and persistent right dorsal aorta. The majority of these anomalies can be identified surgically with a left lateral thoracotomy. However, aberrant right subclavian artery, double aortic arch, or right ductus arteriosus may require an approach via a right lateral thoracotomy [4]. An incorrect surgical approach increases surgical time and morbidity related to postoperative pain and risk of infection. A definitive diagnosis of the type of vascular ring anomaly prior to surgical intervention therefore may decrease morbidity related to prolonged surgical techniques.

A presumptive diagnosis of PRAA is based on historical information and clinical findings. Common radiographic abnormalities that support the diagnosis include enlargement of the cranial mediastinum due to focal megaesophagus, ventral deviation of the trachea cranial to the cardiac silhouette, leftward deviation of the trachea, and absence of the normal left-sided bulge of the ascending aorta $[1,2]$. An esophagram may confirm the presence of focal megaesophagus with evidence of esophageal stricture at the level of the heart base but is unable to delineate vascular structures. Angiography may be useful in detection of atypical vascular rings and may aid in the decision of the best surgical approach [1, 3]. However, it is difficult to discern the three-dimensional anatomic relationships of a vascular ring anomaly with two-dimensional images.

Advanced imaging techniques have improved the ability to definitively diagnose vascular ring anomalies. Computed tomography (CT) and magnetic resonance imaging (MRI) are the imaging modalities of choice in human patients with suspected vascular ring anomalies [5]. CT angiography (CTA) has been used successfully in the diagnosis of vascular ring anomalies in dogs [6-8]. While a diagnosis of a persistent right aortic arch is easily made with CTA, identification of a left ligamentum arteriosum is difficult as blood flow through the ligamentum is rarely present $[1,6,7]$. A similar difficulty was encountered when performing contrast-enhanced MR angiography (CE-MRA) in our patient; 3D reconstructed images of CE-MRA data were less helpful than anticipated due to lack of visualization of nonvascular structures such as trachea and esophagus and lack of blood flow through the ligamentum arteriosum, compromising anatomic orientation. We found delayed postcontrast FLASH 3D images most helpful in evaluating thoracic structures. Diagnosis of persistent right aortic arch with left ligamentum arteriosum and focal megaesophagus was based on identification of the relative positions of the aortic arch, trachea, esophagus, and a small ductus diverticulum. A small soft tissue band thought to represent the ligamentum arteriosum was only indistinctly visible. In contrast, successful identification of a left ligamentum arteriosum with MRI has been documented in a human pediatric case, likely facilitated by larger patient size and a higher magnetic field strength system used [9].

Eight weeks following surgery, the patient was unable to be transitioned to a dry diet. However, the dog was asymptomatic when being fed a mash diet, and the owner was pleased with the outcome. A persistent megaesophagus as in this dog was noted in 13/25 dogs following surgical correction of PRAA [10]. In the same study, $92 \%$ of dogs had complete resolution of clinical signs, whereas $8 \%$ of dogs improved but had occasional episodes of regurgitation.

To the authors' knowledge this is the first report of preoperative confirmation of a PRAA with left ligamentum arteriosum using magnetic resonance angiography in a dog.

\section{Acknowledgments}

This case was presented as a poster at the EVDI Annual Scientific Conference, July 21st-25th 2010, Giessen, Germany.

\section{References}

[1] T. VanGundy, "Vascular ring anomalies," Compendium Small Animal Practice, vol. 11, pp. 36-45, 1989.

[2] J. W. Buchanan, "Tracheal signs and associated vascular anomalies in dogs with persistent right aortic arch," Journal of Veterinary Internal Medicine, vol. 18, no. 4, pp. 510-514, 2004.

[3] M. L. Helphrey, "Vascular ring anomalies in the dog," Veterinary Clinics of North America-Small Animal Practice, vol. 9, no. 2, pp. 207-218, 1979.

[4] G. W. Ellison, "Vascular ring anomalies in the dog and cat," Compendium on Continuing Education for the Veterinary Practitioners, vol. 2, pp. 693-705, 1980.

[5] L. B. Haramati, J. S. Glicksiein, H. J. Issenberg, N. Haramati, and G. A. Crooke, "MR imaging and CT of vascular anomalies and connections in patients with congenital heart disease: significance in surgical planning," Radiographics, vol. 22, no. 2, pp. 337-349, 2002.

[6] S. Pownder and P. V. Scrivani, "Non-selective computed tomography angiography of a vascular ring anomaly in a dog," Journal of Veterinary Cardiology, vol. 10, no. 2, pp. 125-128, 2008.

[7] H. Joly, M. A. D’Anjou, and L. Huneault, "Imaging diagnosis-CT angiography of a rare vascular ring anomaly in a dog," Veterinary Radiology and Ultrasound, vol. 49, no. 1, pp. 42-46, 2008.

[8] C. R. Henjes, I. Nolte, and P. Wefstaedt, "Multidetector-row computed tomography of thoracic aortic anomalies in dogs and cats: patent ductus arteriosus and vascular rings," $B M C$ Veterinary Research, vol. 7, Article ID 57, 2011.

[9] C. H. Zachary, J. L. Myers, and K. D. Eggli, "Vascular ring due to right aortic arch with mirror-image branching and left ligamentum arteriosus: complete preoperative diagnosis by magnetic resonance imaging," Pediatric Cardiology, vol. 22, no. 1, pp. 71-73, 2001.

[10] M. M. Muldoon, S. J. Birchard, and G. W. Ellison, "Long-term results of surgical correction of persistent right aortic in dogs: 25 cases (1980-1995)," Journal of the American Veterinary Medical Association, vol. 210, no. 12, pp. 1761-1763, 1997. 

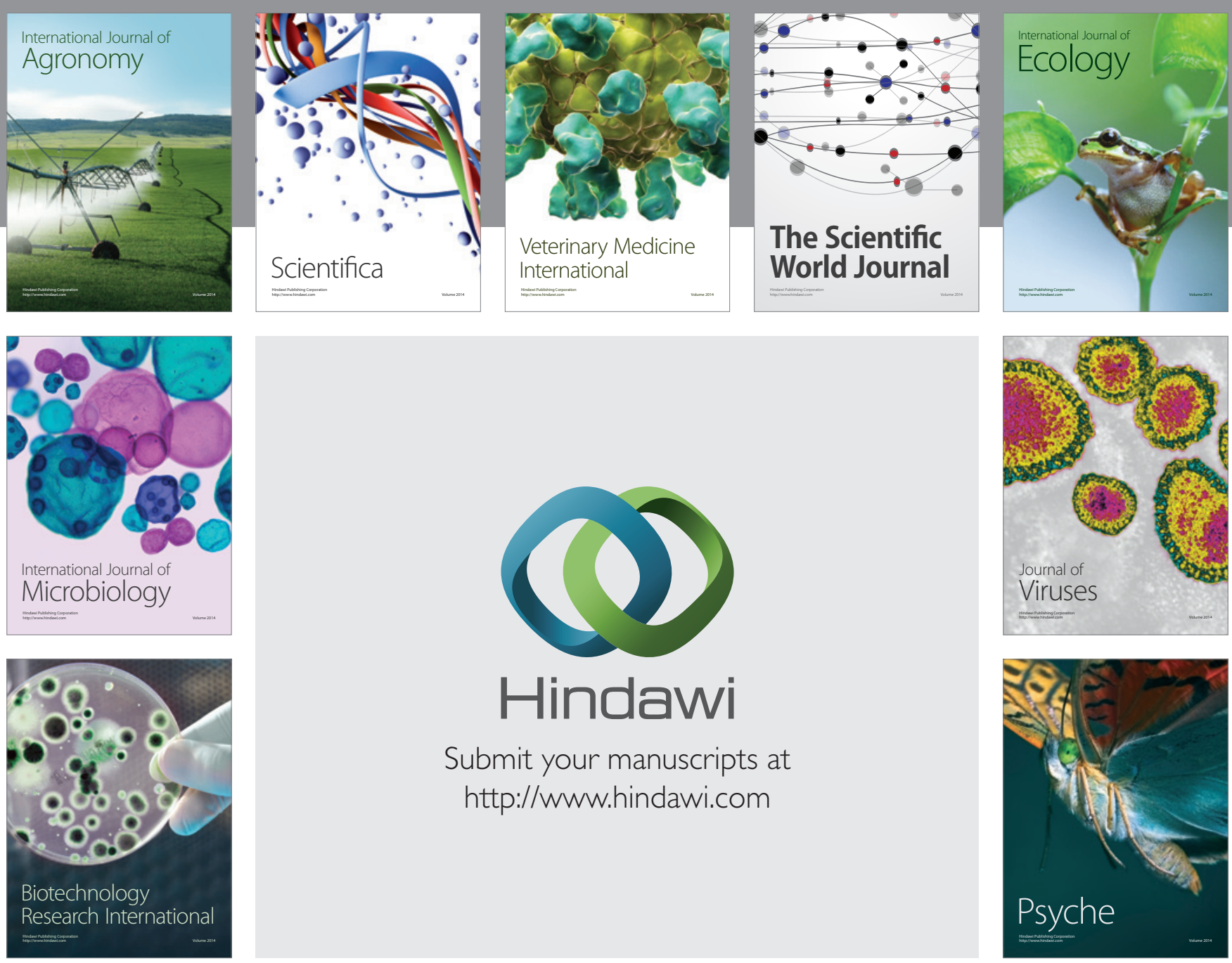

Submit your manuscripts at

http://www.hindawi.com
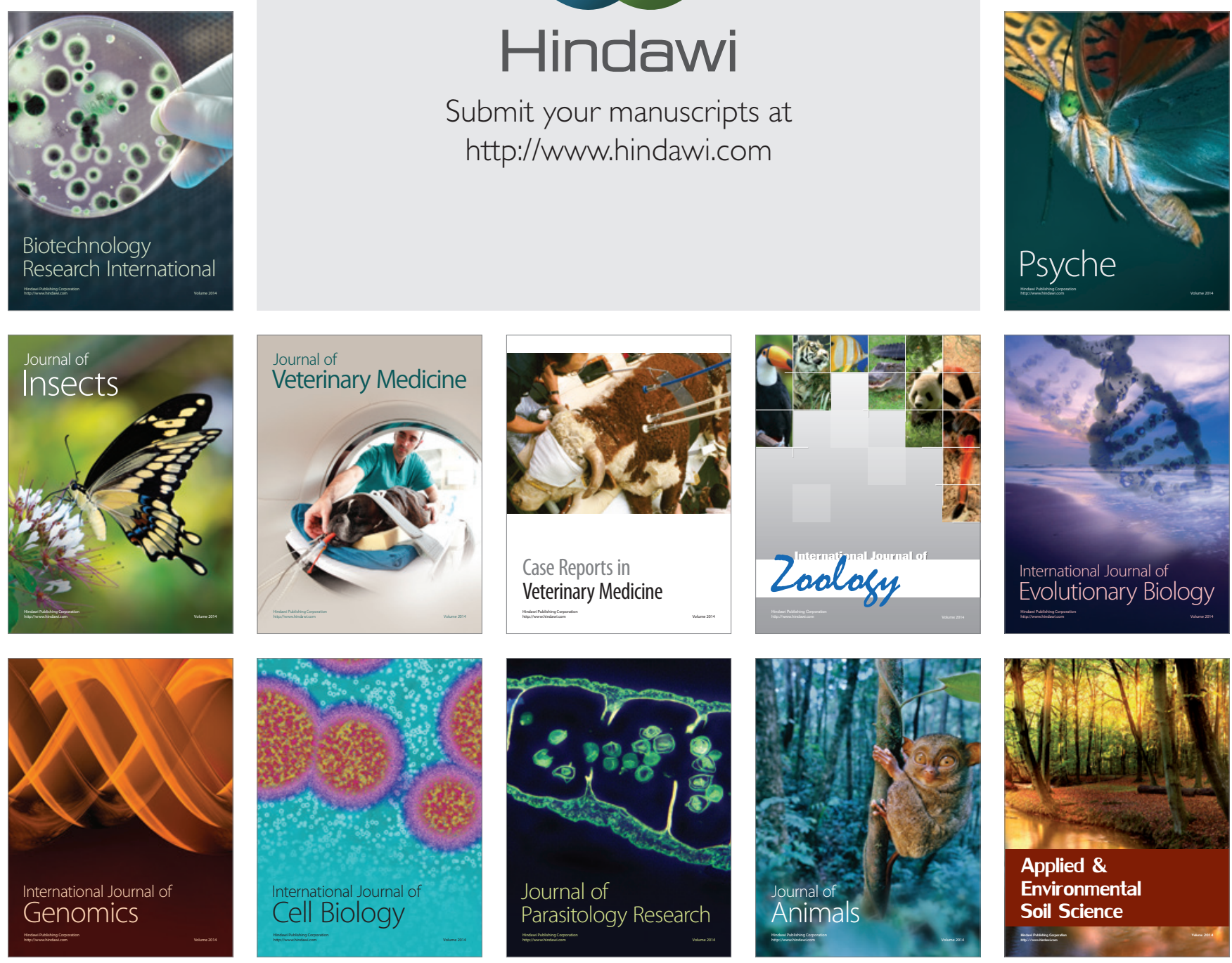